\title{
Knowledge and attitudes on pharmacogenetics among pediatricians
}

\author{
Shahad Rahawi ${ }^{1,2} \cdot$ Hetanshi Naik ${ }^{1} \cdot$ Kathryn V. Blake ${ }^{3,4} \cdot$ Aniwaa Owusu Obeng $^{1,5,6} \cdot$ Rachel M. Wasserman $^{3,4}$. \\ Yoshinori Seki $^{1,2} \cdot$ Vicky L. Funanage ${ }^{3,4} \cdot$ Kimihiko Oishi $^{1,7} \cdot$ Stuart A. Scott $\mathbb{D}^{1,2}$
}

Received: 13 November 2019 / Revised: 6 January 2020 / Accepted: 6 January 2020 / Published online: 27 January 2020

(c) The Author(s), under exclusive licence to The Japan Society of Human Genetics 2020

\begin{abstract}
Increasing enthusiasm for clinical pharmacogenetic testing and the availability of pharmacogenetic-based guidelines indicate that pediatricians will increasingly be expected to interpret and apply pharmacogenetic test results into medical care. Previous studies have identified a lack of knowledge on pharmacogenetics across many physician specialties; however, this has not been systematically assessed among pediatricians. To evaluate pediatrician knowledge, attitude, and educational interest in pharmacogenetics, we surveyed physician cohorts from both the United States (U.S.) and Japan. A total of 282 pediatricians (210 from the U.S. and 72 from Japan) participated in an anonymous survey (online or hardcopy) on pharmacogenetics knowledge, perception, and education. Over $50 \%$ of all respondents had $>10$ years of clinical experience and $>75 \%$ had some prior education in genetics. However, $<10 \%$ felt they were familiar with pharmacogenetics, which was very consistent with $<20 \%$ of the U.S. pediatricians correctly responding to a codeine/CYP2D6 pharmacogenetics knowledge question and $<10 \%$ of U.S. pediatricians being aware of the Clinical Pharmacogenetics Implementation Consortium (CPIC). Despite being generally unfamiliar with pharmacogenetics, $>80 \%$ of all respondents indicated that implementation of clinical pharmacogenetic testing will improve efficacy and safety, and that pediatricians should be capable of applying this testing to their practice. Moreover, the majority $(83.1 \%)$ were interested in educational opportunities on pharmacogenetics, particularly on result interpretation and therapeutic recommendations. Taken together, these data indicate that although practical knowledge of pharmacogenetics among pediatricians in the U.S. and Japan is currently very low, their interest in clinical pharmacogenetics and related education is high, which will likely facilitate future implementation.
\end{abstract}

\section{Introduction}

Supplementary information The online version of this article (https:// doi.org/10.1038/s10038-020-0723-0) contains supplementary material, which is available to authorized users.

Stuart A. Scott

stuart.scott@mssm.edu

1 Department of Genetics and Genomic Sciences, Icahn School of Medicine at Mount Sinai, New York, NY 10029, USA

2 Sema4, Stamford, CT 06902, USA

3 Department of Biomedical Research, Nemours Children's Health System, Wilmington, DE, USA

4 Department of Biomedical Research, Nemours Children's Health System, Jacksonville, Orlando, FL, USA

5 The Charles Bronfman Institute for Personalized Medicine, Icahn School of Medicine at Mount Sinai, New York, NY 10029, USA

6 Department of Pharmacy, The Mount Sinai Hospital, New York, NY 10029, USA

7 Department of Pediatrics, Icahn School of Medicine at Mount Sinai, New York, NY 10029, USA
Clinical pharmacogenetic testing has increasingly become available for healthcare providers, including some academic medical center programs that support pre-emptive pharmacogenetic testing prior to patients being prescribed certain medications [1, 2]. The intent of pre-emptive clinical pharmacogenetic testing is to alert physicians to prescribed medications that have established genetic variants associated with efficacy and/or adverse drug reactions (ADRs). Although most pharmacogenetic programs are currently centered on adult patient populations [1], pharmacogenetic testing will likely have utility for the pediatric population as childhood ADRs account for $\sim 16.5 \%$ of all ADR-related emergency visits in the United States (U.S.) [3]. Moreover, the U.S. Food and Drug Administration (FDA) continues to update drug labels with pharmacogenetic information when indicated, including the recent example of codeine and the increased risks for respiratory depression and death among codeine-treated children who are CYP2D6 ultrarapid metabolizers [4, 5]. 
Despite the growing scientific evidence supporting the clinical validity of specific pharmacogenetic variants and interindividual drug response variability for certain medications, implementing pharmacogenetic-guided prescribing has several challenges, including an inconsistent regulatory and reimbursement landscape, genetic testing turnaround time, a paucity of professional practice guidelines, and inadequate provider education and clinical decision support (CDS) [6-8]. However, evidence-based pharmacogenetic practice guidelines have recently been developed and disseminated for selected gene-drug pairs by the Clinical Pharmacogenetics Implementation Consortium (CPIC; https://cpicpgx. org/guidelines/) [9, 10], which increasingly include specific recommendations for pediatric patients. The availability of CPIC guidelines and the growing number of federally funded clinical research programs dedicated to newborn genome sequencing $[11,12]$ together indicate that physicians, including pediatricians, will increasingly be exposed to pharmacogenetic and other genetic risk factor results.

Given the relatively recent availability of clinical pharmacogenetic testing, healthcare providers generally have reported limited knowledge on pharmacogenetics, which is correlated with a lack of experience in incorporating pharmacogenetic testing into clinical practice [6, 8]. For example, only $\sim 10 \%$ of primary care physicians, cardiologists, and psychiatrists in the U.S. have reported being very or extremely familiar with pharmacogenetics [13, 14]. Given the increasing number of ongoing clinical genome sequencing programs [11], the recent approval of direct-toconsumer commercial pharmacogenetic testing [15], and the paucity of studies on pediatrician perspectives on pharmacogenetics, we sought to evaluate pediatrician knowledge and attitudes on pharmacogenetics in an effort to better inform the design of educational tools on pharmacogenetics.

The feasibility of implementing pharmacogenetic testing will undoubtedly be influenced by local healthcare systems and regulatory agencies, and it may result in diverse perspectives on genetic testing among physicians in different countries. As an initial assessment of international pediatrician perspectives on pharmacogenetics, we also surveyed clinician cohorts from both the U.S. and Japan, acknowledging that significant differences in clinical practice, healthcare systems, and reimbursement models exist between the two countries.

\section{Materials and methods}

\section{Pharmacogenetic survey}

Physician knowledge and attitude questions on pharmacogenetics were adapted from previously reported surveys $[13,14]$. Additional multiple-choice questions included an objective knowledge assessment of the well-characterized codeine/CYP2D6 pharmacogenetic association, previous experience with pharmacogenetic testing, awareness of CPIC and other pharmacogenetic resources, and interest and preferences in pharmacogenetic education. Five-point Likert scale responses were used to report familiarity with pharmacogenetics, helpfulness of revised drug labels, attitudes toward pharmacogenetics, and perceived responsibilities related to testing and results. Open-ended questions were also incorporated into the survey to allow for general comments on pharmacogenetics and the survey (Supplemental Materials 1 and 2).

\section{Provider cohorts}

The anonymous, 20-question survey was sent to pediatricians in the U.S. and Japan through several venues. Email invitations that included a link to the online survey (SurveyMonkey; San Mateo, CA) were deployed through a broadcast service (Medical Marketing Service Inc.; Schaumburg, IL) to 3500 pediatricians in the American Medical Association (AMA) (Supplemental Material 3). In addition, the online survey was emailed to selected pediatrician groups at the Mount Sinai Hospital $(n=\sim 50)$, and clinical pediatricians at the Nemours Children's Health System ( $n=\sim 900$; Supplemental Material 4). Japanese responses were collected through dissemination of a translated survey at professional conferences and through pediatric departments of selected medical institutions $(n=\sim 150)$. The Institutional Review Boards of the Icahn School of Medicine at Mount Sinai and the Nemours Children's Health System deemed this project exempt, and survey responses were collected from December 2017 to March 2018.

\section{Data analysis}

Descriptive statistics summarized the responses to closedended questions, and thematic analysis was performed for open-ended question responses. To facilitate comparisons, pediatricians were classified into "generalists" or "specialists," and Likert scale survey response questions were dichotomized. Chi-square or Fisher's exact tests were performed as appropriate for comparisons between U.S. and Japanese cohorts, and between responses to survey questions of interest. Given the differences in healthcare systems and clinical practice between pediatricians in the U.S. and Japan, only the U.S. cohort was interrogated to assess predictors of familiarity with pharmacogenetics using logistic regression. Univariable analyses were conducted to determine which covariates should be included in the regression model using a threshold $P$ value of $\leq 0.2$. The Japanese cohort sample size did not allow for a regression model analysis. All significance tests were considered 
exploratory and performed at the 0.05 level. Statistical analyses were performed using IBM SPSS Statistics Version 22 (Armonk, NY).

\section{Results}

\section{Subject demographics}

A total of 282 pediatricians, 210 from the U.S. and 72 from Japan, responded to the online survey (Table 1), which

Table 1 Characteristics of survey respondents

\begin{tabular}{|c|c|c|}
\hline Characteristics & U.S. $(n=210)$ & $\begin{array}{l}\text { Japanese } \\
(n=72)\end{array}$ \\
\hline \multicolumn{3}{|l|}{ Years in practice } \\
\hline Currently in training & $5.3 \%(11)$ & $9.7 \%(7)$ \\
\hline $0-4$ & $9.6 \%(20)$ & $6.9 \%(5)$ \\
\hline $5-10$ & $18.8 \%(39)$ & $18.1 \%(13)$ \\
\hline $11-19$ & $23.8 \%(50)$ & $40.3 \%(29)$ \\
\hline $20-29$ & $27.9 \%(58)$ & $16.7 \%(12)$ \\
\hline Over 30 & $15.2 \%(32)$ & $8.3 \%(6)$ \\
\hline \multicolumn{3}{|l|}{ Medical specialty } \\
\hline General pediatrics & $38.1 \%(80)$ & $17.1 \%(12)^{\mathrm{a}}$ \\
\hline Subspecialists & $61.9 \%(130)$ & $83.1 \%(59)^{\mathrm{a}}$ \\
\hline \multicolumn{3}{|l|}{ Practice setting ${ }^{\mathrm{b}}$} \\
\hline $\begin{array}{l}\text { Physician's office, solo } \\
\text { practice }\end{array}$ & $4.8 \%(10)$ & $13.9 \%(10)$ \\
\hline $\begin{array}{l}\text { Physician's office, single } \\
\text { specialty group practice }\end{array}$ & $20.7 \%(43)$ & $5.6 \%(4)$ \\
\hline $\begin{array}{l}\text { Multi-specialty group practice } \\
\text { or clinic }\end{array}$ & $23.6 \%(49)$ & $0 \%(0)$ \\
\hline Hospital, teaching & $55.3 \%(115)$ & $79.2 \%(57)$ \\
\hline Hospital, nonteaching & $4.8 \%(10)$ & $11.1 \%(8)$ \\
\hline Urgent care facility & $1.4 \%(3)$ & $0 \%(0)$ \\
\hline Home health agency & $0 \%(0)$ & $1.2 \%(1)$ \\
\hline \multicolumn{3}{|l|}{ Region } \\
\hline Northeast & $45.7 \%(96)$ & \\
\hline Midwest & $6.7 \%(14)$ & \\
\hline South & $31.0 \%(65)$ & \\
\hline West & $2.4 \%(5)$ & \\
\hline Other & $3.8 \%(8)$ & \\
\hline Unknown & $9.6 \%(20)$ & \\
\hline Tohoku & & $8 \%(6)$ \\
\hline Kanto & & $48.9 \%(35)$ \\
\hline Chubu & & $13 \%(9)$ \\
\hline Shikoku & & $2.8 \%(2)$ \\
\hline Okinawa & & $1.4 \%(1)$ \\
\hline Unknown & & $23.6 \%(17)$ \\
\hline
\end{tabular}

${ }^{\mathrm{a}}$ One respondent did not answer

'This question was "select all that apply" resulted in an overall response rate of $\sim 6.1 \%(282 / \sim 4600)$. However, the estimated response rates from the AMA, Nemours, and Japanese cohorts ranged from 0.8 to $\sim 50 \%$ (Supplemental Material 5). Over 50\% of respondents had more than ten years of experience. General pediatricians accounted for $32.7 \%$ of respondents (92/281). Most pediatricians practiced one or more subspecialties; however, these varied across 33 subspecialties, the most frequent among the combined U.S. and Japanese cohort being medical genetics $(12.2 \%, 23 / 189)$, hematology oncology $(11.1 \%, 21 / 189)$, and neonatal-perinatal medicine $(10.6 \%$, 20/189) (Supplemental Material 6).

\section{Knowledge assessment on pharmacogenetics}

Less than $10 \%$ of all respondents reported being very familiar with pharmacogenetics (Fig. 1). As expected, medical geneticists were more likely to be familiar with pharmacogenetics compared with all other respondents $(p<0.001)$. Approximately $12.0 \%$ (3/25) of pediatricians who had been practicing for $0-4$ years indicated they were familiar with pharmacogenetics, which was a higher frequency than reported by pediatricians with more years of experience (7.8\%; 20/256).

An objective assessment of pediatrician knowledge on codeine pharmacogenetics determined that over half of respondents did not know which pharmacogenetic metabolizer phenotype increases the risk for respiratory depression and death among children treated with codeine $(63.7 \%$, $179 / 281)$. However, it is important to note that codeine is not commonly used in pediatric practice in Japan (see "Discussion"). The correct answer ("CYP2D6 ultrarapid metabolizers") was selected by 41/281 respondents (18.6\%) (Fig. 1). Anesthesiologists and emergency medicine physicians selected the correct answer more than other specialists.

\section{Familiarity with pharmacogenetics}

Interestingly, $19.6 \%(51 / 260)$ of respondents indicated that they had previously ordered a clinical pharmacogenetic test, with U.S. specialists being significantly more likely to have ordered a test compared with U.S. generalists $(p=0.013)$. In the U.S cohort, a significant predictor of familiarity with pharmacogenetics was awareness of CPIC (Table 2). However, only $7.2 \%(20 / 277)$ of responding pediatricians were aware of CPIC pharmacogenetic practice guidelines (Fig. 1). Furthermore, only $38.5 \%$ (80/208) of U.S. pediatricians were aware that several FDA drug labels currently include pharmacogenetic information to help guide therapeutic management. Out of the 74 providers who were aware of drug label pharmacogenetic information, only $39.1 \%$ (29/74) stated that the information was "somewhat helpful" and only $14.9 \%$ (11/74) reported them as "very" or "extremely helpful." 


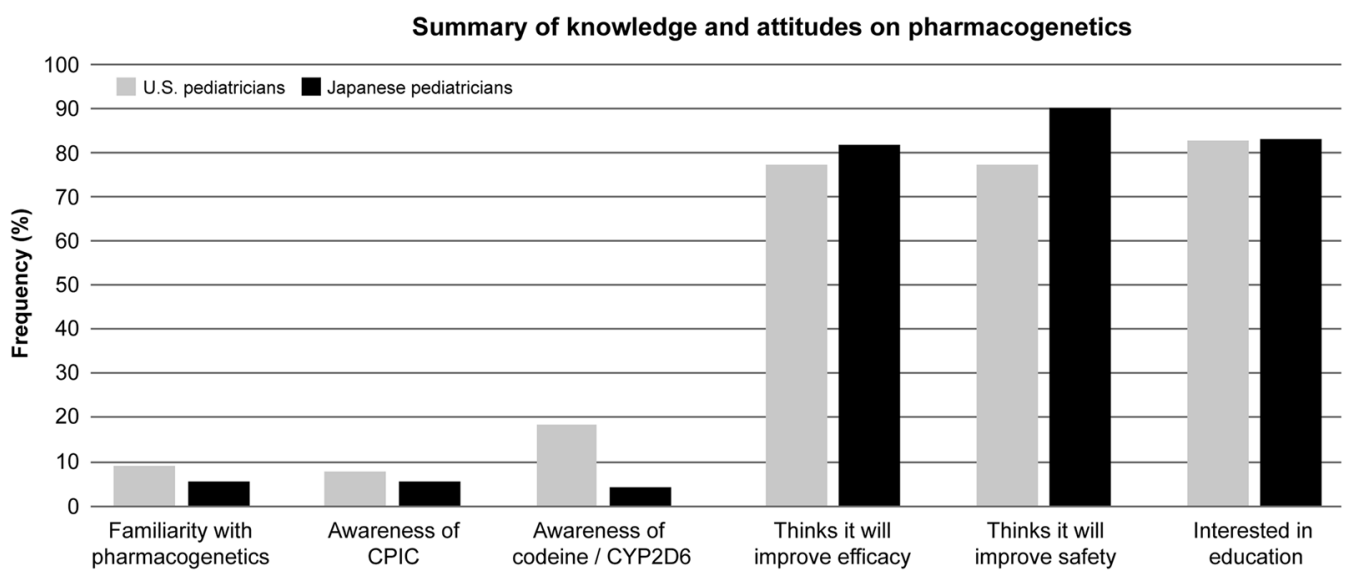

Fig. 1 Summary of pediatrician knowledge and attitudes on pharmacogenetics. Illustrated are the graphical results for six central components of the survey: self-reported familiarity with pharmacogenetics; awareness of the Clinical Pharmacogenetics Implementation
Consortium (CPIC); knowledge of the codeine/CYP2D6 pharmacogenetic association; perception of role of pharmacogenetics in drug efficacy; perception of role of pharmacogenetics in drug safety; and pediatrician interest in pharmacogenetic education
Table 2 Logistic regression model for familiarity with pharmacogenetics among pediatricians in the U.S.

\begin{tabular}{|c|c|c|c|}
\hline & $\beta$ Estimate & $\begin{array}{l}\text { Adjusted OR } \\
(95 \% \mathrm{Cl})\end{array}$ & $P$ value \\
\hline \multicolumn{4}{|l|}{ Familiarity with pharmacogenetics $\left(n=195^{\mathrm{a}}\right)$} \\
\hline Specialists vs. generalists & -0.07 & $0.93(0.25,3.5)$ & 0.91 \\
\hline Awareness of CPIC vs. not & 2.2 & $9.2(2.3,36)$ & 0.001 \\
\hline $\begin{array}{l}\text { Correctly identified codeine CYP2D6 metabolizer } \\
\text { phenotype vs. not }\end{array}$ & 0.55 & $1.7(0.50,6.0)$ & 0.39 \\
\hline $\begin{array}{l}\text { Awareness of pharmacogenetics in FDA drug labels } \\
\text { vs. not }\end{array}$ & 1.2 & $3.3(0.92,12)$ & 0.066 \\
\hline Previously ordered a pharmacogenetics test vs. not & 0.92 & $2.5(0.78,8.1)$ & 0.12 \\
\hline
\end{tabular}

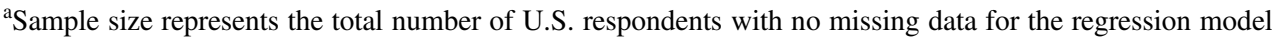

\section{Attitudes on pharmacogenetics}

Less than 20\% (47/268) of pediatricians felt they were well informed about the role of clinical pharmacogenetic testing in therapeutic decision-making; however, $>80 \%$ of providers considered it a valuable tool to both improve drug efficacy $(81.7 \%, 219 / 268)$ and predict the risk of adverse events (84.0\%, 225/268) (Fig. 1). Few respondents felt that pharmacogenetics is too difficult for pediatricians to understand $(14.8 \%, 39 / 264)$. Importantly, the majority of all respondents indicated that pediatricians should hold pivotal responsibilities when it comes to pharmacogenetic testing, including being able to identify medications that require pharmacogenetic testing $(84.6 \%, 225 / 266)$, discuss testing and results with patients $(85.7 \%, 227 / 265)$, apply these results to alter prescribing $(84.5 \%, 224 / 265)$, and identify informational sources for providers and patients $(91.4 \%, 244 / 267)$.

\section{Pharmacogenetics education preferences}

Despite a general lack of familiarity with pharmacogenetics, $89.3 \%(233 / 261)$ of all respondents expressed interest in professional educational offerings on pharmacogenetics (Fig. 1). Of those not interested in pharmacogenetics education, the most commonly cited reasons were time constraints $(47.6 \%, 10 / 21)$ and inapplicability to their medical specialty $(28.6 \%, 6 / 21)$ (e.g., radiology). When asked what resources are currently used to answer questions about pharmacogenetics, pediatricians cited scientific literature (77.7\%, 219/282), the internet (e.g., Google, WebMD, UpToDate) $(58.9 \%, 166 / 282)$, and medical guidelines $(58.9 \%, 166 / 282)$. Of note, only $11.7 \%$ (33/282) of pediatricians selected consultation of PharmGKB or CPIC, and even fewer indicated that they consulted pharmacists $(7.1 \%$, 20/282).

Pediatricians were interested in educational offerings in the format of grand rounds $(53.9 \%, 124 / 230), \mathrm{CME} / \mathrm{CE}$ courses $(55.7 \%, 128 / 230)$, and seminars or lectures $(64.8 \%$, 149/230) (Table 3). U.S. and Japanese pediatricians favored learning about recommendations for prescribing depending on pharmacogenetic results $(85.7 \%, 197 / 230)$, interpretation of pharmacogenetic test results $(78.7 \%, 181 / 230)$, and the effect of genetic variation on drug mechanism of action $(70.0 \%, 161 / 230)$. Thematic analysis suggested that the 
Table 3 Preferred characteristics of a pharmacogenetic educational resource

\begin{tabular}{|c|c|c|}
\hline Characteristics & U.S. $(n=172)$ & $\begin{array}{l}\text { Japanese } \\
(n=58)\end{array}$ \\
\hline \multicolumn{3}{|l|}{ Education content } \\
\hline $\begin{array}{l}\text { Recommendations (if any) for } \\
\text { prescribing }\end{array}$ & $91.2 \%(156)$ & $70.7 \%(41)$ \\
\hline $\begin{array}{l}\text { How to interpret } \\
\text { pharmacogenetic test results }\end{array}$ & $81.9 \%(140)$ & $70.7 \%(41)$ \\
\hline $\begin{array}{l}\text { Effect of genetic variation on } \\
\text { mechanism of drug action }\end{array}$ & $70.2 \%(120)$ & $70.7 \%(41)$ \\
\hline $\begin{array}{l}\text { Description of pharmacogenetic } \\
\text { information in drug labeling }\end{array}$ & $63.2 \%(108)$ & $60.3 \%(35)$ \\
\hline $\begin{array}{l}\text { Demographics of populations } \\
\text { likely to carry genetic } \\
\text { variations }\end{array}$ & $68.4 \%(117)$ & $53.4 \%(31)$ \\
\hline $\begin{array}{l}\text { Laboratories offering } \\
\text { pharmacogenetic testing }\end{array}$ & $47.4 \%(81)$ & $50.0 \%(29)$ \\
\hline References (scientific literature) & $35.7 \%(61)$ & $43.1 \%(25)$ \\
\hline \multicolumn{3}{|l|}{ Education format } \\
\hline Seminar or lecture & $55.6 \%(95)$ & $93.1 \%(54)$ \\
\hline Web-based CME/CE course & $61.4 \%(105)$ & $39.7 \%(23)$ \\
\hline Journal articles & $49.7 \%(85)$ & $32.8 \%(19)$ \\
\hline Grand rounds & $62.6 \%(107)$ & $29.3 \%(17)$ \\
\hline $\mathrm{CME} / \mathrm{CE}$ course & $60.8 \%(104)$ & $24.1 \%(14)$ \\
\hline AHEC course & $1.8 \%(3)$ & $15.5 \%(9)$ \\
\hline Half-day conference & $21.1 \%(36)$ & $12.1 \%(7)$ \\
\hline All-day conference & $15.8 \%(27)$ & $12.1 \%(7)$ \\
\hline
\end{tabular}

majority of the responders who selected "other" stated that they prefer to learn about cost and ordering of tests.

\section{Comparison of U.S. and Japanese pediatrician responses}

General pediatricians accounted for $38.1 \%$ and $17.1 \%$ of U. S. and Japanese respondents, respectively $(p<0.001)$. U.S. pediatricians were most frequently "somewhat familiar" with pharmacogenetics $(45.9 \%, 96 / 209)$, whereas Japanese pediatricians were most frequently "not familiar" $(36.1 \%$, 25/72). As such, U.S. pediatricians were significantly more likely to select the correct codeine pharmacogenetics answer than respondents from Japan $\left(X^{2}(1, N=281)=\right.$ $8.85, p=0.003)$; however, it is important to note that pediatric codeine usage is more common in the U.S. than Japan. In addition, U.S. pediatricians were significantly more likely to have ordered a test $\left(X^{2}(1, N=270)=5.47\right.$, $p=0.019)$ compared with Japanese pediatricians. Few U.S. pediatricians $(8.2 \%, 16 / 196)$ felt that pharmacogenetics is too difficult for pediatricians to understand, while a higher percentage of Japanese pediatricians $(33.8 \%, 23 / 68)$ agreed with that sentiment. Regarding educational preferences, the Japanese pediatricians displayed more interest in attending seminars or lectures $(93.1 \%, 54 / 58)$ than their American counterparts $(55.6 \%, 95 / 172)$ (Table 3$)$.

\section{Discussion}

Given the increasing enthusiasm for implementing pharmacogenetic-guided medication prescribing and the availability and expansion of genome sequencing programs for both adults and children, we sought to determine pediatrician knowledge and attitudes on pharmacogenetics to better inform their educational needs related to clinical pharmacogenetic testing. Our results clearly demonstrate that familiarity and knowledge of pharmacogenetics among pediatricians currently is very low, as $<10 \%$ of survey respondents indicated that they felt very or extremely familiar. However, this was contrasted by their high interest in clinical pharmacogenetics and related education, as $>80 \%$ of all respondents indicated that pediatricians should hold pivotal responsibilities and competency when it comes to clinical pharmacogenetic testing. Although pediatricians do typically coordinate and oversee all aspects of patient care, they specifically acknowledged through the survey that they should be able to identify medications that require pharmacogenetic testing, interpret and apply testing results, discuss results with patients, and identify reliable sources of information on pharmacogenetics for both providers and patients.

The level of knowledge on pharmacogenetics reported among pediatricians in our study is consistent with previous reports that surveyed primary care physicians, cardiologists, psychiatrists, and other physician specialties [8, 13, 14]. The self-reported lack of familiarity on pharmacogenetics among pediatricians was corroborated by direct assessment of their knowledge, as only $18.3 \%$ of U.S. pediatricians selected the correct answer when asked about the pharmacogenetic association between CYP2D6 and codeine response. However, U.S. pediatricians specializing in anesthesiology and emergency medicine chose the correct answer at a higher frequency than other specialties, indicating that pharmacogenetics knowledge may correlate with specialty. This is consistent with previous studies that reported more cardiologists correctly identified clopidogrel as having a genotype-dependent response variability compared with primary care physicians and psychiatrists [14].

Importantly, survey respondents indicated that they considered pharmacogenetics a valuable tool to improve both drug efficacy and safety. The survey did not directly allow respondents to explain why they believed pharmacogenomics would improve their practice; however, our assumption is that pediatricians largely believe that germline pharmacogenetic variation has clinical validity and/or utility with respect to interindividual response differences 
that they have observed among their patient populations. To this end, the majority ( $80 \%)$ of pediatricians also expressed that they should be capable of performing duties related to pharmacogenetic testing, including patient education, test ordering, and communication of test results. However, the data from our study and others $[8,13,14,16]$ clearly indicates that a significant disparity exists across providers between their current understanding of pharmacogenetics and the level of knowledge needed to support these services. Pharmacogenetic test ordering, interpretation, and result communication can also be supported by pharmacists and genetic counselors in the U.S. [17]; however, given the only recent availability of clinical pharmacogenetic testing, both of these professions have also reported an educational need for pharmacogenetic competency [16, 18].

A commonly cited challenge by providers and medical centers when considering clinical pharmacogenetic testing is a need for professional practice guidelines [7, 19-21]. In an effort to address this vital component, the CPIC was formed in 2009 to develop and disseminate evidence-based guidelines for pharmacogenetic-guided therapy without any formal recommendation for or against testing $[9,10]$. These published guidelines typically are centered around specific gene-drug pairs, and to date are publicly available for 19 genes and $>45$ medications. Moreover, the majority of CPIC guidelines now include specific recommendations for pediatric prescribing; however, $<10 \%$ of U.S. pediatrician respondents in our study indicated that they were aware of the CPIC. This result directly speaks to the need for broader dissemination of CPIC guidelines and related resources to practicing physicians and their professional societies. Similarly, the majority $(\sim 60 \%)$ of U.S. pediatricians were unaware that some FDA drug labels included pharmacogenetic information, which is consistent with data from previous studies [8, 13]. Although the pharmacogenetic information included in some FDA drug labels is often localized to the pharmacokinetics and pharmacodynamics subsection, these survey results further support the need for provider education on pharmacogenetics and highlight the challenges with translating FDA label pharmacokinetic information into specific dosing recommendations.

To assess potential differences in international physician perspectives on pharmacogenetics, our survey was also deployed to pediatricians in Japan, who in general have lower accessibility to clinical genetic testing compared with pediatricians in the U.S [22]. However, several progressive efforts on pharmacogenomics have been established in Japan. For example, mandatory insurance-covered pharmacogenetic testing of UGTIAl prior to initiation of irinotecan chemotherapy has been in place since 2008. The Japan Society of Human Genetics, Japanese Society of Clinical Pharmacology and Therapeutics, and Japanese Committee for Clinical Laboratory Standards subsequently published clinical pharmacogenetic guidelines in 2010 [23]. Moreover, the Japan Pharmacogenomics Data Science Consortium was established in 2009 by six pharmaceutical companies (Astellas, Daiichi Sankyo, Mitsubishi Tanabe, Otsuka, Taisho, and Takeda) with the aim of supporting pharmacogenomic discovery in Japan during drug development and post-market [24]. Consistent with these efforts, $81.0 \%$ of the general Japanese public previously reported a positive attitude towards pharmacogenetics research [25]. As such, it is notable that our survey study identified a similar lack of knowledge on pharmacogenetics among Japanese pediatricians compared with the U.S. cohort. Interestingly, physicians from the U.S. and Japan both agreed on the ranking or order of importance for the specific content of a pharmacogenetic educational resource but differed in the preferred format(s) in which it is presented (Table 3). To our knowledge, this is the first study that assessed the knowledge and attitudes of Japanese physicians on pharmacogenetics.

However, it is important to note that major differences in clinical practice exist between the U.S. and Japan, including medication prescribing patterns (e.g., codeine), drug labeling and language, clinical pharmacist roles and responsibilities, and medical reimbursement systems. As such, any differences in pediatrician perspectives identified between the two countries should acknowledge these potential sources of confounding. For example, physician prescribing patterns are likely very influenced by local regulatory body oversight, as well as the degree of exposure to pharmacogenetic information in drug labels. In fact, more Japanese pediatricians felt that pharmacogenetics is too difficult to understand and showed more interest in learning opportunities, suggesting a deficiency of exposure to pharmacogenetics in their routine clinical settings compared with their U.S. counterparts. As such, future surveys with questions on multiple drugs that are commonly prescribed in each country may more effectively measure differences in pharmacogenetic knowledge between international pediatrician cohorts.

Although the majority of participating pediatricians indicated that they received some genetics education during their medical training, it is very unlikely that pharmacogenetics was included given that the majority completed medical school $\geq 10$ years prior to the survey. As medical schools evolve to incorporate pharmacogenetics into their curricula [26], the continuing advances in the field of pharmacogenetics and the availability of CPIC and FDA recommendations strongly suggest that additional and ongoing educational resources are needed for practicing clinicians. To directly inform the design of those educational instruments, our study determined that the preferred educational resource for pediatricians should focus on therapeutic decision recommendations, testing logistics 
(ordering and costs), and the integration of CDS into their workflow and the electronic medical record. Importantly, several pre-emptive clinical pharmacogenetic testing programs are currently ongoing at selected medical centers with the aim of assessing the feasibility and clinical adoption of CDS-based pharmacogenetic-guided prescribing [1, 27-29].

Limitations of this study include a modest sample size with a geographic distribution and range of experience that may limit the generalizability of results. More experienced physicians completed the survey compared with recent graduates, and previous studies have found that experienced physicians more readily adopt genetic technologies [8]; however, no statistical difference in familiarity was detected in our cohort between pediatricians in earlier or later years of their career. In addition, the Japanese survey was directly translated from the English survey, which could have led to unaccounted for bias within the responses from the Japanese respondents. The Japanese Pharmaceutical and Medical Device agency oversees drug labels and is equivalent to the U.S. FDA but was not mentioned in the Japanese translation. Other potential confounding between the U.S. and Japanese cohorts is mentioned above; however, it is also noteworthy that there currently are very few Japanese members in the CPIC (https://cpicpgx.org), which would likely translate to reduced awareness. In addition, our sample of respondents may be biased towards those more interested in pharmacogenetic testing and more inclined to respond to a survey on the topic, leading to a potential overestimation of knowledge and/or interest in our study. Lastly, given that our survey focused on cohorts from two developed countries, an assessment across multiple countries would enable a more generalizable evaluation of pediatrician knowledge and attitudes on pharmacogenetics.

In conclusion, our study is the first reported survey of pediatrician knowledge and attitudes on clinical pharmacogenetics. These novel results clearly indicate that although pediatricians currently have a low level of familiarity with pharmacogenetics, the majority perceive it to be an effective tool for enhancing drug efficacy and safety. This study also underscores the importance of developing educational resources on clinical pharmacogenetics for pediatricians, including the availability of therapeutic recommendations and related support, which taken together may facilitate clinical implementation.

Acknowledgements The authors would like to thank Stacey Gray, from the Nemours Biomedical Research Department, for coordinating the administration of the Nemours physician survey.

Conflict of interest SR, YS, and SAS are paid employees of Sema4.

Publisher's note Springer Nature remains neutral with regard to jurisdictional claims in published maps and institutional affiliations.

\section{References}

1. Dunnenberger HM, Crews KR, Hoffman JM, Caudle KE, Broeckel U, Howard SC, et al. Preemptive clinical pharmacogenetics implementation: current programs in five US medical centers. Annu Rev Pharm Toxicol. 2015;55:89-106.

2. Rasmussen-Torvik LJ, Stallings SC, Gordon AS, Almoguera B, Basford MA, Bielinski SJ, et al. Design and anticipated outcomes of the eMERGE-PGx project: a multicenter pilot for preemptive pharmacogenomics in electronic health record systems. Clin Pharm Ther. 2014;96:482-9.

3. Shehab N, Lovegrove MC, Geller AI, Rose KO, Weidle NJ, Budnitz DS. US Emergency Department visits for outpatient adverse drug events, 2013-2014. J Am Med Assoc. 2016;316: 2115-25.

4. Chidambaran V, Sadhasivam S, Mahmoud M. Codeine and opioid metabolism: implications and alternatives for pediatric pain management. Curr Opin Anaesthesiol. 2017;30:349-56.

5. Hudak ML. Codeine pharmacogenetics as a proof of concept for pediatric precision medicine. Pediatrics. 2016;138. pii: e20161359.

6. Ieiri I. What are barriers to pharmacogenomics (PGx) clinical uptake? Drug Metab Pharmacokinet. 2012;27:279.

7. Scott SA. Personalizing medicine with clinical pharmacogenetics. Genet Med. 2011;13:987-95.

8. Stanek EJ, Sanders CL, Taber KA, Khalid M, Patel A, Verbrugge $\mathrm{RR}$, et al. Adoption of pharmacogenomic testing by US physicians: results of a nationwide survey. Clin Pharm Ther. 2012;91:450-8.

9. Relling MV, Klein TE. CPIC: Clinical Pharmacogenetics Implementation Consortium of the pharmacogenomics research network. Clin Pharm Ther. 2011;89:464-7.

10. Relling MV, Klein TE, Gammal RS, Whirl-Carrillo M, Hoffman JM, Caudle KE. The Clinical Pharmacogenetics Implementation Consortium: 10 years later. Clin Pharmacol Ther. 2020;107:171-5.

11. Berg JS, Agrawal PB, Bailey DB, Jr., Beggs AH, Brenner SE, Brower AM, et al. Newborn sequencing in genomic medicine and public health. Pediatrics. 2017;139. pii: e20162252.

12. Holm IA, McGuire A, Pereira S, Rehm H, Green RC, Beggs AH, et al. Returning a genomic result for an adult-onset condition to the parents of a newborn: insights from the BabySeq project. Pediatrics. 2019;143:S37-43.

13. Haga SB, Burke W, Ginsburg GS, Mills R, Agans R. Primary care physicians' knowledge of and experience with pharmacogenetic testing. Clin Genet. 2012;82:388-94.

14. Johansen Taber KA, Dickinson BD. Pharmacogenomic knowledge gaps and educational resource needs among physicians in selected specialties. Pharmgenomics Pers Med. 2014;7:145-62.

15. Lu M, Lewis CM, Traylor M. Pharmacogenetic testing through the direct-to-consumer genetic testing company 23andMe. BMC Med Genomics. 2017;10:47.

16. Haga SB, O'Daniel JM, Tindall GM, Mills R, Lipkus IM, Agans R. Survey of genetic counselors and clinical geneticists' use and attitudes toward pharmacogenetic testing. Clin Genet. 2012;82:115-20.

17. Mills R, Haga SB. Clinical delivery of pharmacogenetic testing services: a proposed partnership between genetic counselors and pharmacists. Pharmacogenomics. 2013;14:957-68.

18. McCullough KB, Formea CM, Berg KD, Burzynski JA, Cunningham JL, Ou NN, et al. Assessment of the pharmacogenomics educational needs of pharmacists. Am J Pharm Educ. 2011;75:51.

19. Amstutz U, Carleton BC. Pharmacogenetic testing: time for clinical practice guidelines. Clin Pharm Ther. 2011;89:924-7.

20. Relling MV, Evans WE. Pharmacogenomics in the clinic. Nature. 2015;526:343-50.

21. Zineh I, Lesko LJ. Pharmacogenetics in medicine: barriers, critical factors and a framework for dialogue. Per Med. 2009;6:359-61. 
22. Watanabe M, Ohata T, Muto K, Takada F. Problems in the regulation of genetic tests in Japan: what can we learn from direct-toconsumer genetic tests? Public Health Genomics. 2010;13: 327-35.

23. Suematsu K. Japan Pharmacogenomics Data Science Consortium database and its application for drug safety analyses. Curr Dermatol Rep. 2016;5:1-4.

24. Kamitsuji S, Matsuda T, Nishimura K, Endo S, Wada C, Watanabe K, et al. Japan PGx Data Science Consortium Database: SNPs and HLA genotype data from 2994 Japanese healthy individuals for pharmacogenomics studies. J Hum Genet. 2015;60: 319-26.

25. Kobayashi E, Sakurada T, Ueda S, Satoh N. Public involvement in pharmacogenomics research: a national survey on patients' attitudes towards pharmacogenomics research and the willingness to donate DNA samples to a DNA bank in Japan. Cell Tissue Bank. 2011;12:71-80.
26. Green JS, O’Brien TJ, Chiappinelli VA, Harralson AF. Pharmacogenomics instruction in US and Canadian medical schools: implications for personalized medicine. Pharmacogenomics. 2010; 11:1331-40.

27. Hoffman JM, Haidar CE, Wilkinson MR, Crews KR, Baker DK, Kornegay NM, et al. PG4KDS: a model for the clinical implementation of pre-emptive pharmacogenetics. Am J Med Genet C Semin Med Genet. 2014;166C:45-55.

28. Luzum JA, Pakyz RE, Elsey AR, Haidar CE, Peterson JF, WhirlCarrillo $\mathrm{M}$, et al. The pharmacogenomics research network translational pharmacogenetics program: outcomes and metrics of pharmacogenetic implementations across diverse healthcare systems. Clin Pharm Ther. 2017;102:502-10.

29. Bell GC, Crews KR, Wilkinson MR, Haidar CE, Hicks JK, Baker DK, et al. Development and use of active clinical decision support for preemptive pharmacogenomics. J Am Med Inf Assoc. 2014;21:e93-9. 